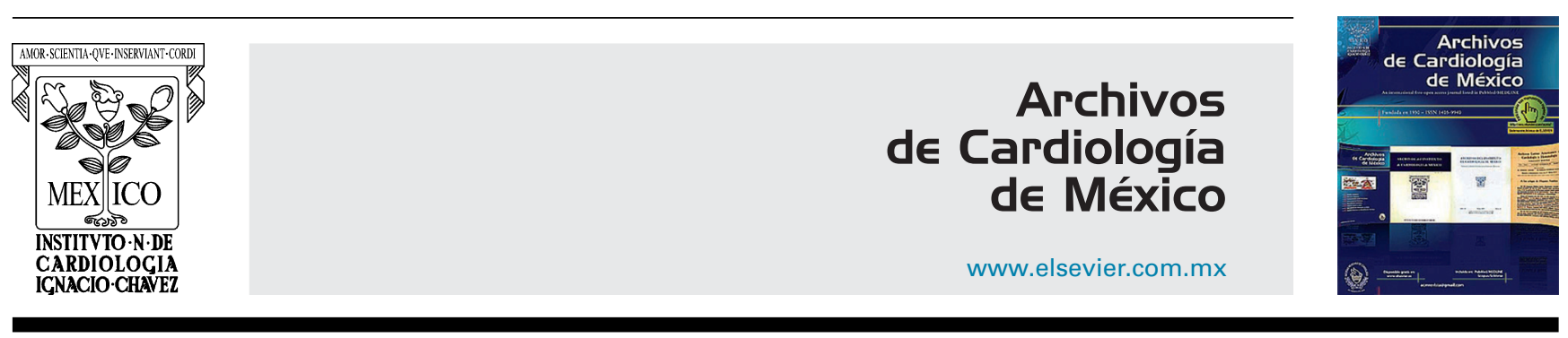

CARTAS CIENTÍFICAS

\section{Trombosis valvular protésica tratada con infusión lenta de fibrinolíticos en paciente con accidente cerebrovascular isquémico agudo}

\section{Prosthetic heart valve thrombosis treated with slow infusion fibrinolytic therapy in patient with acute isquemic stroke}

La trombosis de válvula protésica mecánica es una seria complicación, dada su alta tasa de mortalidad, que oscila entre el $80-90 \%$. Su incidencia varía de acuerdo a las diferentes series, encontrándose entre el $0.3 \%$ y el $1.3 \%$ por año ${ }^{1}$.

Los factores de riesgo más importantes para el desarrollo de la misma son: la posición mitral de la prótesis y una anticoagulación en subrango terapéutico ${ }^{2}$.

Este cuadro puede manifestarse clínicamente como disfunción valvular protésica (que ocasiona insuficiencia cardiaca o arritmias) o embolias sistémicas. El ecocardiograma transesofágico (ETE) es el método de referencia para el diagnóstico de disfunción protésica ${ }^{3}$.

Las opciones terapéuticas son: la anticoagulación, la trombectomía quirúrgica y la trombólisis sistémica ${ }^{4}$. En líneas generales, las guías de valvulopatías, tanto europeas como americanas, recomiendan la cirugía de urgencia cuando el paciente se encuentra descompensado hemodinámicamente, con disnea de reposo, o cuando el trombo es móvil y tiene un tamaño mayor a $10 \mathrm{~mm}$. Por el contrario, la terapia fibrinolítica se reserva para los pacientes estables hemodinámicamente con disnea de esfuerzo o cuando la cirugía de urgencia no está disponible, o el paciente es de alto riesgo quirúrgico ${ }^{5-7}$.

Por último, es importante resaltar que las características anatómicas de la válvula halladas en el procedimiento de reemplazo valvular podrán condicionar el abordaje terapéutico posterior.

Sin embargo, la estrategia terapéutica en pacientes que cursan con un accidente cerebrovascular isquémico secundario a trombosis valvular mecánica protésica es controversial.

\section{Informe de caso}

Presentamos el caso de un paciente de sexo masculino de 54 años de edad que consultó a la guardia de nuestro hospital refiriendo hemiparesia derecha. El paciente tenía antecedentes de fibrilación auricular persistente y reemplazo valvular mitral (estenosis mitral reumática) con prótesis mecánica un mes atrás.

El paciente se hallaba anticoagulado con antagonistas de la vitamina $\mathrm{K}$.

Al momento de la admisión, sus signos vitales eran: tensión arterial, 118/70 mmHg; frecuencia cardiaca, 83 latidos por minuto; frecuencia respiratoria, 18 por minuto; saturación de oxígeno $98 \%$ aire ambiente; temperatura axilar de $36.6^{\circ} \mathrm{C}$. El examen neurológico reveló como datos positivos: debilidad braquiocrural derecha $4 / 5$, con disminución de los reflejos osteotendinosos en los 4 miembros, escala del National Institute of Health Stroke Scale (NIHSS): 3 puntos. El examen cardiovascular no evidenció signos de insuficiencia cardiaca ni soplos audibles.

Luego de la cirugía, el paciente no logra un tiempo en rango terapéutico adecuado.

Se realizó tomografía computada y resonancia magnética nuclear de cerebro que evidenciaron un infarto agudo en territorio de la arteria cerebral anterior izquierda (fig. $1 \mathrm{~A}$ y B).

El ETE evidenció 2 imágenes móviles compatibles con trombos del lado auricular de la prótesis mitral, que prolapsaban hacia el ventrículo izquierdo en diástole (fig. 2 A y B).

A las $48 \mathrm{~h}$, se realizó una tomografía computada de control que descartó transformación hemorrágica, por lo que se inició anticoagulación con heparina no fraccionada intravenosa, rotando luego a enoxaparina. El paciente evolucionó clínicamente estable, sin nuevos síntomas. Al octavo día del ingreso se realiza un nuevo ETE que mostró crecimiento del trombo, alcanzando un diámetro máximo de $15 \mathrm{~mm}$, con importante movilidad transvalvular.

El caso fue discutido en forma multidisciplinaria, entre cardiólogos, cirujanos cardiovasculares y neurólogos. Debido a la extensa calcificación a nivel del aparato valvular observado al momento del reemplazo valvular, con riesgo inadmisible de rotura del surco auriculoventricular, se decide optar a pesar de un riesgo de transformación 


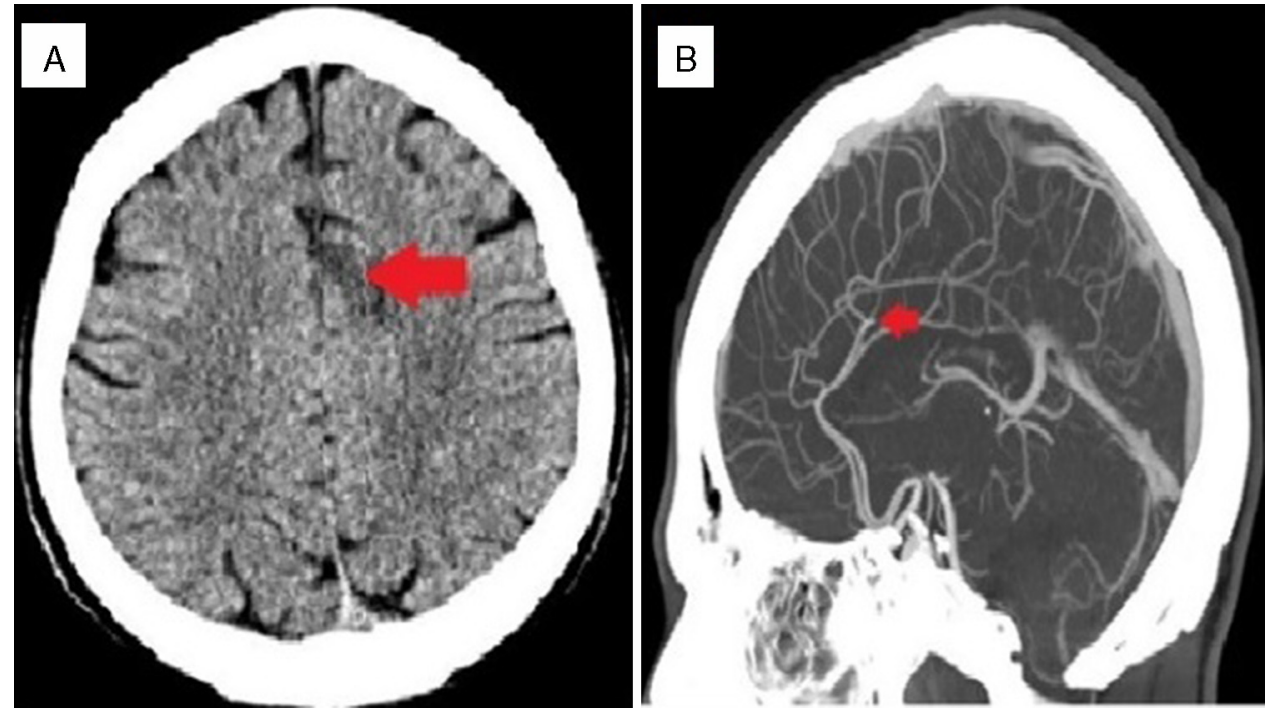

Figura 1 (A) Se observa una lesión hipodensa corticosubcortical en el sector posterior de la circunvolución del cíngulo del lado izquierdo, compatible con infarto agudo en territorio de la arteria cerebral anterior correspondiente (flecha). (B) Angiotomografía de vasos intracraneales evidencia compromiso de arteria cerebral anterior izquierda con lesión oclusiva a nivel del ramo A3 (flecha).
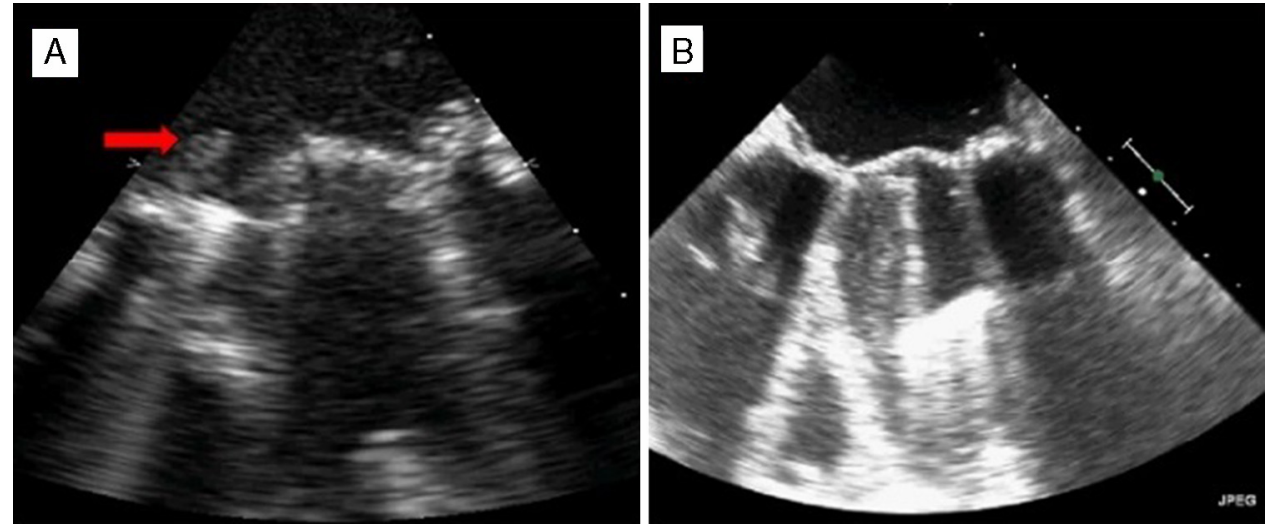

Figura 2 (A) Ecocardiograma bidimensional transesofágico a nivel medioesofágico permite observar a nivel posteroseptal sobre la cara auricular del anillo protésico 2 imágenes hipoecogénicas, móviles, una de $1.25 \mathrm{~cm}$ de largo (flecha) y otra de $0.6 \mathrm{~cm}$ de largo, compatibles con trombo, a correlacionar con el cuadro clínico. (B) Ecocardiograma bidimensional luego de infusión de fibrinolítico donde se evidencia ausencia de imagen trombótica.

hemorrágica intracraneal no menor (10\%) y/o fragmentación del trombo (20\%) por el tratamiento con fibrinolíticos sistémicos.

Se administró estreptocinasa a razón de 500,000 UI en $20 \mathrm{~min}$, y 1,500,000 en infusión continua lenta en $10 \mathrm{~h}$. El paciente no presentó ninguna complicación. Tanto el tiempo de protrombina, KPTT y recuento plaquetario se mantuvieron en valores normales luego de la infusión salvo una marcada hipofibrinogenemia con valores por debajo de 100 . Luego de efectuar una tomografía computada de cerebro de control a las $24 \mathrm{~h}$ sin evidencia de sangrado se decide iniciar enoxaparina $0.7 \mathrm{mg} / \mathrm{kg} / 12 \mathrm{~h}$.

Se efectúa un ETE a las $48 \mathrm{~h}$ de la trombólisis revelando disolución del trombo. Sumado a un dosaje de fibrinógeno mayor a 200 se decide aumentar la administración de enoxaparina a dosis de $1 \mathrm{mg} / \mathrm{kg} / 12 \mathrm{~h}$.

Al cuarto día se rota anticoagulación a antagonistas de la vitamina $\mathrm{K}$, logrando rango terapéutico en 6 días. Dada la buena evolución se otorga el alta hospitalaria prescribiéndole aspirina en bajas dosis sumado a anticoagulación con warfarina indicando un objetivo de RIN de 4.

\section{Discusión}

La trombosis valvular mecánica protésica es una rara complicación del reemplazo valvular, pero con una alta tasa de morbimortalidad. El diagnóstico precoz es fundamental en el curso de esta enfermedad, dado que una terapéutica temprana exitosa mejora el pronóstico a corto y largo plazo.

El riesgo de embolismo en las trombosis de válvula protésica mecánica depende del tamaño y de la movilidad del trombo, teniendo un riesgo mayor al $80 \%$ los trombos mayores a $8 \mathrm{~mm}^{8}$.

El perfil de seguridad de la cirugía es superior a la fibrinólisis en pacientes que cursan con un accidente cerebrovascular isquémico. La terapia fibrinolítica posee 
mayor riesgo de sangrado (10-15\%) y de embolismo periférico (20-30\%) debido a fragmentación. Sin embargo, Özkan et al. reportaron que dosis bajas e infusiones lentas de fibrinolíticos eran no inferiores a las dosis estándar, y que el perfil de seguridad era mayor, con menores tasas de sangrado y embolismo?.

Como mencionamos previamente, la cirugía fue desestimada por cuestiones técnicas, optándose por la fibrinólisis en infusión lenta luego de evidenciar una ausencia de respuesta favorable a la anticoagulación con heparina no fraccionada en rango adecuado durante una semana.

El protocolo fibrinolítico elegido fue estreptocinasa a razón de 500,000 Ul en 20 min seguido por la infusión continua lenta de 1,500,000 Ul en $10 \mathrm{~h}$. Sin embargo, existen otros esquemas utilizados y descriptos en la literatura en estudios observacionales:

- Alteplasa $10 \mathrm{mg}$ en bolo seguido por $90 \mathrm{mg}$ en infusión continua durante $120 \mathrm{~min}$.

- Alteplasa $20 \mathrm{mg}$ en bolo seguido por $30 \mathrm{mg}$ en infusión continua durante $180 \mathrm{~min}$.

- Alteplasa $25 \mathrm{mg}$ en infusión continua durante $6 \mathrm{~h}$ sin administración de bolo previo.

En casos de inestabilidad hemodinámica se recomienda un protocolo corto, ya sea R-TPA $10 \mathrm{mg}$ en bolo $+90 \mathrm{mg}$ en infusión continua por $120 \mathrm{~min}$ asociado a heparina no fraccionada; o estreptocinasa $1,500,000 \mathrm{UI}$ en 60 minutos sin heparina no fraccionada. Infusiones más prolongadas pueden ser utilizadas en pacientes estables ${ }^{1}$.

Un esquema de R-TPA más bajo usando $20 \mathrm{mg}$ en bolo $+10 \mathrm{mg}$ por hora en infusión continua por $3 \mathrm{~h}$ puede ser utilizado en algunas situaciones.

Ha sido descripto un menor rédito de la fibrinólisis sistémica en situaciones como trombosis protésica en posición mitral, trombosis crónicas o presencia de pannus, muchas veces indistinguible del trombo ${ }^{10}$.

\section{Conclusión}

Este es el primer caso reportado en la literatura acerca del uso exitoso de la terapia fibrinolítica en pacientes con trombosis de válvula protésica mecánica, en contexto de un accidente cerebrovascular.

Consideramos este reporte una contribución al manejo de estos pacientes donde la estrategia de acción a la fecha sigue siendo controversial.

\section{Bibliografía}

1. Roudaut R, Serri K, Lafitte S. Thrombosis of prosthetic heart valves: Diagnosis and therapeutic considerations. Heart. 2007;93:137-42.

2. Gohlke-Barwolf C. Anticoagulation in valvar heart disease: New aspects and management during non-cardiac surgery. Heart. 2000;84:567-72.

3. Gueret $P$, Vignon $P$, Fournier $P$, et al. Transesophageal echocardiography for the diagnosis and management of nonobstructive thrombosis of mechanical mitral valve prosthesis. Circulation. 1995;91:103-10.
4. Shapira Y, Herz I, Vaturi M, et al. Thrombolysis is an effective and safe therapy in stuck bileaflet mitral valves in the absence of high-risk thrombi. J Am Coll Cardiol. 2000;35: 1874-80.

5. Roudaut R, Lafitte S, Roudaut MF, et al. Management of prosthetic heart valve obstruction: Fibrinolysis versus surgery. Early results and long-term follow-up in a single-centre study of 263 cases. Arch Cardiovasc Dis. 2009;102:269-77.

6. Toker ME, Eren E, Balkanay M, et al. Multivariate analysis for operative mortality in obstructive prosthetic valve dysfunction due to pannus and thrombus formation. Int Heart J. 2006:47:237-45.

7. Whitlock RP, Sun JC, Fremes SE, et al., American College of Chest Physicians. Antithrombotic and thrombolytic therapy for valvular disease: Antithrombotic therapy and prevention of thrombosis, 9th ed: American College of Chest Physicians Evidence-Based Clinical Practice Guidelines. Chest. 2012;141 2 Suppl:e576S-600S.

8. Ramos Al, Ramos RF, Togna DJ, et al. Fibrinolytic therapy for thrombosis in cardiac valvular prosthesis short and long term results. Arq Bras Cardiol. 2003;81:393-8, 387-392.

9. Özkan $M$, Gündüz S, Biteker $M$, et al. Comparison of different TEE-guided thrombolytic regimens for prosthetic valve thrombosis: The TROIA trial. JACC Cardiovasc Imaging. 2013;6:206-16

10. Zoghbi WA, Chambers JB, Dumesnil JG, et al. Recommendations for evaluation of prosthetic valves with echocardiography and doppler ultrasound: A report from the American Society of Echocardiography's Guidelines and Standards Committee and the Task Force on Prosthetic Valves, developed in conjunction with the American College of Cardiology Cardiovascular Imaging Committee, Cardiac Imaging Committee of the American Heart Association, the European Association of Echocardiography, a registered branch of the European Society of Cardiology, the Japanese Society of Echocardiography and the Canadian Society of Echocardiography, endorsed by the American College of Cardiology Foundation, American Heart Association, European Association of Echocardiography, a registered branch of the European Society of Cardiology, the Japanese Society of Echocardiography, and Canadian Society of Echocardiography. J Am Soc Echocardiogr. 2009;22:975-1014, quiz 1082-1014.

Fernando Garagoli a,*, Francisco José Romeo ${ }^{a}$, Luciano Lucas ${ }^{\mathrm{a}}$, Vadim Kotowicz ${ }^{\mathrm{b}}$ y César Belziti ${ }^{\mathrm{a}}$

\section{a Servicio de Cardiología, Hospital Italiano de Buenos Aires, Ciudad Autónoma Buenos Aires, Argentina b Servicio de Cirugía Cardiovascular, Hospital Italiano de Buenos Aires, Ciudad Autónoma Buenos Aires, Argentina}

* Autor para correspondencia. Hospital Italiano de Buenos Aires. Unidad Coronaria. Perón 4190. Capital Federal Código Postal C1199ABB. Teléfono: +541149590200 , interno 8920/8921; fax: +541149590200, interno 8758 . Correo electrónico:

Fernando.garagoli@hospitalitaliano.org.ar (F. Garagoli).

http://dx.doi.org/10.1016/j.acmx.2016.05.012

1405-9940/

(c) 2016 Instituto Nacional de Cardiología Ignacio Chávez. Publicado por Masson Doyma México S.A. Este es un artículo Open Access bajo la CC BY-NC-ND licencia (http://creativecommons.org/ licencias/by-nc-nd/4.0/). 